\title{
Defibrotide in preventing veno-occlusive disease after hematopoietic stem cell transplantation in children with osteopetrosis
}

\author{
Justyna Miśkiewicz-Bujna , Izabella Miśkiewicz-Migoń, Monika Rosa, Karolina Liszka, \\ Krzysztof Kałwak
}

Department of Pediatric Bone Marrow Transplantation, Oncology and Hematology, Wrocław Medical University, Wrocław, Poland

A 5-month-old girl diagnosed with osteopetrosis (OP) caused by homozygous mutation in the SNX10 gene was admitted to the Department of Pediatric Bone Marrow Transplantation, Oncology and Hematology in Wroclaw, Poland for high-dose chemotherapy with subsequent allogeneic hematopoietic stem cell transplantation (allo-HSCT).

The infant was born at gestational age 41 weeks with Apgar score 10. Antenatal period was uneventful. The newborn's hearing screening test revealed no abnormalities. The patient's family history was notable for osteopetrosis confirmed in a brother who had died aged 4 . An older sister was healthy. In the course of diagnostics, normocytic anemia with reticulocytosis, and elevated levels of ferritin, lactate dehydrogenase (LDH), creatine kinase-myocardial bound (CK-MB) and vitamin A were found. Myelogram showed reduced cellularity of bone marrow in each lineage, with absence of megakaryocytes. Bone X-ray showed an increase in bone sclerosis with blurred trabeculae structure, but no pathologic fractures were detected. The conditioning regimen consisted of busulfan intravenous (i.v.) $(4 \times 4 \times 1 \mathrm{mg} / \mathrm{kg})$, fludarabine $\left(5 \times 30 \mathrm{mg} / \mathrm{m}^{2}\right)$ and thiotepa $(2 \times 5 \mathrm{mg} / \mathrm{kg})$. Cyclosporin A was implemented for the prevention of graft rejection and graft versus host disease (GvHD) from day -1 before hematopoietic stem cell transplantation (HSCT). Ursodeoxycholic acid was administered from day -12 as the routine prevention of hepatic veno-occlusive disease (VOD). The patient received prophylactic defibrotide (off-label) $(25 \mathrm{mg} / \mathrm{kg} /$ day) from day +2 after HSCT onwards. Bone marrow transplantation from her 10/10 humen leukocyte antigen (HLA)-matched sister was performed. $6.81 \times 10^{6} \mathrm{CD} 34^{+}$cells/kg were infused.
On day +20 , hepatomegaly was diagnosed by palpation of the abdomen with the detection of the liver $3.5 \mathrm{~cm}$ below costal margin and then confirmed by abdominal ultrasound (enlarged liver size approximately $8.1 \mathrm{~cm}$ with increased echogenicity and features of steatosis without focal changes). Weight gain requiring implementation of furosemide, increased consumption of platelets with normalization after weight-adjusted platelet substitution, rising bilirubin from a baseline value up to $1.4 \mathrm{mg} / \mathrm{dl}$ with fast normalization, decreased antithrombin III activity, and enlarged abdominal circumference were observed.

The patient was diagnosed with mild VOD based on the European Society for Blood and Marrow Transplantation (EBMT) criteria (Table I) due to the presence of the following: weight gain $>5 \%$ above baseline value; and hepatomegaly confirmed by ultrasound [1]. She was also treated with spironolactone from day +20 for two days with drug withdrawal due to hyperkaliemia and hyponatremia. Defibrotide was implemented prophylactically on day +2 , and was continued during the VOD course. No side effects of long-term therapy with defibrotide were observed during hospitalization. The patient's condition began to improve with normalization of bilirubin level and weight, and hepatomegaly resolution. VOD symptoms resolved on day +27 , and defibrotide was stopped on day +30 thereafter. The child was discharged on day +41 without significant adverse events.

A 2-year follow-up revealed no neurological symptoms, and the psychomotor development of the girl was satisfactory. No late hepatic VOD was observed.

Osteopetrosis is a genetic disorder with heterogeneous clinical phenotypes, characterized by excessively high

\footnotetext{
*Address for correspondence: Justyna Miśkiewicz-Bujna, Department of Pediatric Bone Marrow Transplantation, Oncology and Hematology, Wrocław Medical University, Wybrzeże Ludwika Pasteura 1, Wrocław 50-367, Poland, e-mail: miskiewicz.justyna@gmail.com

\section{PTHiT Copyright $\odot 2021$}

The Polish Society of Haematologists and Transfusiologists, Insitute of Haematology and Transfusion Medicine. All rights reserved. 
Table I. European Society for Blood and Marrow Transplantation (EBMT) diagnostic criteria for hepatic sinusoidal obstruction syndrome/veno-occlusive disease (SOS/VOD) in children [1]

No limitation for time of onset of SOS/VOD

The presence of two or more of the following*:

- unexplained consumptive and transfusion-refractory thrombocytopenia**

- otherwise unexplained weight gain on three consecutive days despite use of diuretics or weight gain $>5 \%$ above baseline value

- hepatomegaly*** (best if confirmed by imaging) above baseline value

- ascites ${ }^{* *}$ (best if confirmed by imaging) above baseline value

- bilirubin rising from baseline value or bilirubin $\geq 2 \mathrm{mg} / \mathrm{dL}$ within $72 \mathrm{~h}$

*With the exclusion of other potential differential diagnoses; ** $\geq 1$ weight-adjusted platelet substitution/day to maintain institutional transfusion guidelines; ***suggested: imaging [ultrasound (US), computed tomography (CT) or magnetic resonance imaging (MRI)] immediately before hematopoietic cell transplantation (HCT) to determine baseline value for both hepatomegaly and ascites

bone density caused by defects in the formation or function of osteoclasts [2]. Three forms of osteopetrosis have been described: autosomal recessive osteopetrosis (ARO), autosomal dominant osteopetrosis, and X-linked osteopetrosis [3]. The diagnosis of ARO, the most severe of the three forms, is made soon after birth. Left untreated it is lethal, with a mortality rate of $70 \%$ by age 6 [4]. OP requires a distinct strategy and personalized management. Therapeutic options are limited, and HSCT remains the only life-saving method. HSCT should be performed as soon as possible in children with OP, preferably before the age of 10 months [2], thereby preventing irreversible complications regarding vision, hearing and/or hematopoiesis dysfunction [5]. Recipients of HLA-identical HSCT appear to have a 5 -year disease-free survival of approximately $73 \%$ [6]. Patients with ARO have a higher incidence of VOD after HSCT than do children with other underlying diseases (63.6\% vs. $10-30 \%$ ) [7]. Prophylaxis of VOD includes implementation of ursodeoxycholic acid and defibrotide (off-label) [8]. Defibrotide adverse effects include diarrhea, nausea, vomiting, epistaxis, hypotension, pulmonary alveolar hemorrhage, cerebral hemorrhage and sepsis [9]. Deciding which patients should receive defibrotide as VOD prophylaxis remains the subject of debate.

In describing this case, we intended to emphasize the importance of this off-label prophylactic method in this specific cohort of patients. In our Department, we adapted the expert recommendations of Corbacioglu et al. [7] and gave off-label defibrotide at $25 \mathrm{mg} / \mathrm{kg} /$ day as a prophylaxis of VOD after HSCT. In the described patient, only a mild form of VOD developed and the child did not require treatment in a pediatric intensive care unit.
The outcome of our patient confirms the conclusion of Corbacioglu et al. [10] controlled trial in which the rate of VOD was lower in patients with OP receiving defibrotide prophylaxis compared to controls.

In conclusion, off-label VOD prophylaxis with defibrotide significantly reduced the severity of VOD in our patient, and ensured a mild post-transplant course and swift discharge home. The benefits clearly outweighed the possible complications of using prophylactic defibrotide.

VOD is a complication which increases the risk of transplant-related mortality, as well as affecting the quality of life of a child. Therefore, the implementation of prophylactic defibrotide before HSCT seems reasonable and fully justified in this high-risk group of patients.

\section{References}

1. Corbacioglu S, Carreras E, Ansari M, et al. Diagnosis and severity criteria for sinusoidal obstruction syndrome/veno-occlusive disease in pediatric patients: a new classification from the European society for blood and marrow transplantation. Bone Marrow Transplant. 2017; 53(2): 138-145, doi: 10.1038/bmt.2017.161, indexed in Pubmed: 28759025.

2. Penna S, Capo V, Palagano E, et al. One disease, many genes: implications for the treatment of osteopetroses. Front Endocrinol (Lausanne). 2019; 10: 85, doi: 10.3389/fendo.2019.00085, indexed in Pubmed: 30837952.

3. Palagano E, Menale C, Sobacchi C, et al. Genetics of osteopetrosis. Curr Osteoporos Rep. 2018; 16(1): 13-25, doi: 10.1007/s11914018-0415-2.

4. Orchard PJ, Fasth AL, Le Rademacher J, et al. Hematopoietic stem cell transplantation for infantile osteopetrosis. Blood. 2015; 126(2): 270-276, doi: 10.1182/blood-2015-01-625541, indexed in Pubmed: 26012570.

5. Shadur B, Zaidman I, NaserEddin A, et al. Successful hematopoietic stem cell transplantation for osteopetrosis using reduced intensity conditioning. Pediatr Blood Cancer. 2018; 65(6): e27010, doi: 10.1002/pbc.27010, indexed in Pubmed: 29469225.

6. Driessen GJA, Gerritsen EJA, Fischer A, et al. Long-term outcome of haematopoietic stem cell transplantation in autosomal recessive osteopetrosis: an EBMT report. Bone Marrow Transplant. 2003; 32(7): 657-663, doi: 10.1038/sj.bmt.1704194, indexed in Pubmed: 13130312.

7. Corbacioglu S, Hönig M, Lahr G, et al. Stem cell transplantation in children with infantile osteopetrosis is associated with a high incidence of VOD, which could be prevented with defibrotide. Bone Marrow Transplant. 2006; 38(8): 547-553, doi: 10.1038/sj.bmt.1705485, indexed in Pubmed: 16953210.

8. Qureshi A, Marshall L, Lancaster D. Defibrotide in the prevention and treatment of veno-occlusive disease in autologous and allogeneic stem cell transplantation in children. Pediatr Blood Cancer. 2008; 50(4): 831-832, doi: 10.1002/pbc.21425, indexed in Pubmed: 18286502.

9. Baker DE, Demaris K. Defibrotide. Hosp Pharm. 2016; 51(10): $847-$ -854, doi: 10.1310/hpj5110-847, indexed in Pubmed: 27928191.

10. Corbacioglu S, Schulz A, Sedlacek P, et al. Defibrotide for prophylaxis of hepatic veno-occlusive disease in pediatric hematopoietic stem cell transplantation: subanalysis data from an open-label, phase III, randomized trial. Blood. 2015; 126(23): 4310-4310, doi: 10.1182/ /blood.v126.23.4310.4310. 